\title{
In Situ Transmission Electron Microscopy Study of Electron Beam-Induced Transformations in Colloidal Cesium Lead Halide Perovskite Nanocrystals
}

Zhiya Dang, ${ }^{\dagger}$ Javad Shamsi, $^{\dagger \dagger}$ Francisco Palazon, ${ }^{\dagger}$ Muhammad Imran, ${ }^{\dagger, \dagger}$ Quinten A. Akkerman, ${ }^{\dagger,}$ Sungwook Park, ${ }^{\dagger, \S}$ Giovanni Bertoni, ${ }^{\dagger,}{ }^{\circ}$ Mirko Prato, ${ }^{\dagger}$ Rosaria Brescia, ${ }^{*},{ }^{\dagger}$ and Liberato Manna ${ }^{*}, \dagger$

${ }^{\dagger}$ Department of Nanochemistry, Istituto Italiano di Tecnologia, Via Morego 30, 16163 Genova, Italy

${ }^{\ddagger}$ Dipartimento di Chimica e Chimica Industriale, Università degli Studi di Genova, Via Dodecaneso, 31, 16146 Genova, Italy

${ }^{\S}$ Department of Physics, Pukyong National University, Busan 608-737, Korea

"IMEM-CNR, Parco Area delle Scienze 37/A, Parma 43124, Italy

Supporting Information

ABSTRACT: An increasing number of studies have recently reported the rapid degradation of hybrid and allinorganic lead halide perovskite nanocrystals under electron beam irradiation in the transmission electron microscope, with the formation of nanometer size, high contrast particles. The nature of these nanoparticles and the involved transformations in the perovskite nanocrystals are still a matter of debate. Herein, we have studied the effects of high energy $(80 / 200 \mathrm{keV})$ electron irradiation on colloidal cesium lead bromide $\left(\mathrm{CsPbBr}_{3}\right)$ nanocrystals with
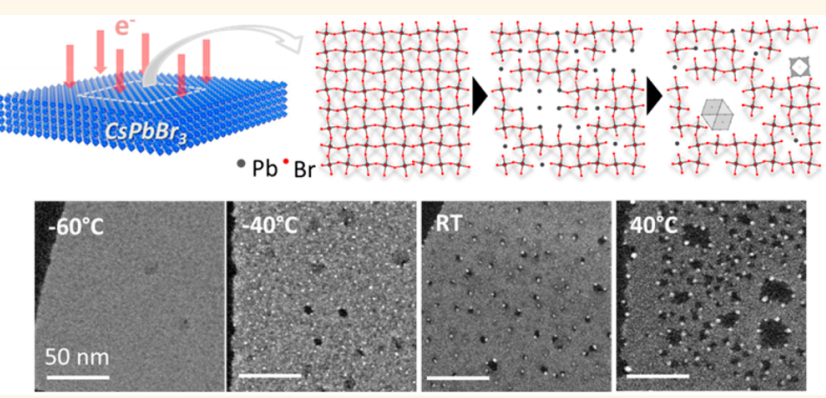
different shapes and sizes, especially $3 \mathrm{~nm}$ thick nanosheets, a morphology that facilitated the analysis of the various ongoing processes. Our results show that the $\mathrm{CsPbBr}_{3}$ nanocrystals undergo a radiolysis process, with electron stimulated desorption of a fraction of bromine atoms and the reduction of a fraction of $\mathrm{Pb}^{2+}$ ions to $\mathrm{Pb}^{0}$. Subsequently $\mathrm{Pb}^{0}$ atoms diffuse and aggregate, giving rise to the high contrast particles, as previously reported by various groups. The diffusion is facilitated by both high temperature and electron beam irradiation. The early stage $\mathrm{Pb}$ nanoparticles are epitaxially bound to the parent $\mathrm{CsPbBr}_{3}$ lattice, and evolve into nonepitaxially bound $\mathrm{Pb}$ crystals upon further irradiation, leading to local amorphization and consequent dismantling of the $\mathrm{CsPbBr}_{3}$ lattice. The comparison among $\mathrm{CsPbBr}_{3}$ nanocrystals with various shapes and sizes evidences that the damage is particularly pronounced at the corners and edges of the surface, due to a lower diffusion barrier for $\mathrm{Pb}^{0}$ on the surface than inside the crystal and the presence of a larger fraction of under-coordinated atoms.

KEYWORDS: cesium lead halide nanocrystals, TEM, radiation damage

$\mathrm{L}$ ead halide perovskites, both hybrid and all-inorganic, with general formula $\mathrm{APbX}_{3}$ (with $\mathrm{A}^{+}=\mathrm{CH}_{3} \mathrm{NH}_{3}^{+}$/ $\mathrm{NH}_{2} \mathrm{CH}=\mathrm{NH}_{2}^{+} / \mathrm{Cs}^{+}$, and $\left.\mathrm{X}^{-}=\mathrm{Cl}^{-} / \mathrm{Br}^{-} / \mathrm{I}^{-}\right)$, have remarkable electronic properties ${ }^{1}$ that make them promising for applications in photovoltaics ${ }^{2}$ and optoelectronics. ${ }^{3-5}$ Compared to their bulk counterparts, $\mathrm{APbX}_{3}$ nanocrystals (NCs) have one or more dimensions that are reduced to a few to tens of nanometers, and their size and morphology can be manipulated to tune their optical properties. ${ }^{6,7}$ However, since surfaces and interfaces play important roles in material degradation, ${ }^{8,9}$ perovskite NCs may be inherently more unstable under irradiation by several sources (laser light, $\mathrm{X}$ rays, electrons), heating, or exposure to moisture, than the corresponding bulk crystals. ${ }^{6-10}$ Such instability represents a major limitation for the widespread application of halide perovskite NCs in devices.

Recent transmission electron microscopy (TEM) studies of lead halide perovskite $\mathrm{NCs}\left(\mathrm{CH}_{3} \mathrm{NH}_{3} \mathrm{PbX}_{3},{ }^{10,11}\right.$ $\left(\mathrm{C}_{4} \mathrm{H}_{9} \mathrm{NH}_{3}\right)_{2} \mathrm{PbBr}_{4}{ }^{12}$ and $\left.\mathrm{CsPbX}_{3}{ }^{13-17}\right)$ have consistently reported the appearance of few nanometer size high contrast particles upon irradiation. Some of these reports indicated that the particles are made of metallic lead, ${ }^{11,13,16,17}$ a claim that is

Received: December 12, 2016

Accepted: January 25, 2017

Published: January 25, 2017 
supported by the evidence that $\mathrm{Pb}^{2+}$ species from various material systems can be reduced to $\mathrm{Pb}^{0}$ by irradiation with electrons ${ }^{18}$ or with X-rays. ${ }^{8,19}$ Other studies of irradiation of $\mathrm{APbX}_{3} \mathrm{NCs}$ have suggested that $\mathrm{PbBr}_{2}{ }^{11}$ is also formed. Such discrepancies among the various reports underline an incomplete understanding of the overall transformations in the perovskite NCs, and especially of those leading to these high contrast particles. Compared to halide perovskites, the behavior of other materials systems under electron beam irradiation is better understood. For example, previous studies on oxides and on $\mathrm{CaF}_{2}$ have shown that their irradiation by an electron beam can induce the nucleation of metal particles and surface metallization. ${ }^{20-24}$ One typical case is the transformation of $\mathrm{PbO}_{2}$ to $\mathrm{Pb}$ through an intermediate $\mathrm{PbO}$ phase. ${ }^{18}$ It is known that the nucleation of metal particles and the surface metallization on oxides or on $\mathrm{CaF}_{2}$ occur in parallel with electron stimulated desorption processes through electronic excitation: in practice, while the cations are reduced to their metallic states, the anions (i.e., oxygen ions in oxides or fluoride ions in $\mathrm{CaF}_{2}$ ) are oxidized to neutral or even to positive species, which readily desorb from the surface. ${ }^{21,25-27}$ Stimulated desorption processes also occur under other types of irradiation, for example by photons. ${ }^{25}$

Here we demonstrate that high energy $(80 / 200 \mathrm{keV})$ electron irradiation of $\mathrm{APbX}_{3}\left(\mathrm{CsPb}_{3}\right.$ in the present case) NCs in a TEM causes the desorption of halogen species and the nucleation of metallic $\mathrm{Pb}$ particles. Also, by a temperature dependent study we were able to provide several details of the mechanism by which the $\mathrm{Pb}$ nanoparticles are formed. Our analysis was carried out on colloidal NCs having different shapes (cubes, wires, sheets), although it was mainly focused on $\mathrm{CsPbBr}_{3}$ nanosheets with $3 \mathrm{~nm}$ thickness and hundreds of $\mathrm{nm}$ width, a morphology that allows the observation of multiple events under identical irradiation conditions. We could identify two main stages in the transformation: (i) electron stimulated desorption of $\mathrm{Br}$ atoms and concomitant reduction of $\mathrm{Pb}^{2+}$ to $\mathrm{Pb}^{0}$, and (ii) diffusion of $\mathrm{Pb}^{0}$ atoms and their aggregation into $\mathrm{Pb}$ nanoparticles, with local amorphization and severe dismantling of the $\mathrm{CsPbBr}_{3}$ NCs. This process is more pronounced at lower electron energy, higher electron dose, and higher temperature, and is favored in higher surface-tovolume ratio NCs.

\section{RESULTS AND DISCUSSION}

Knock-on damage and radiolysis are the two major electronspecimen interactions operative in an electron microscope with an incident electron energy of tens or hundreds of kiloelectron volt $(\mathrm{keV}) .^{28}$ Knock-on damage involves an irreversible displacement of the nuclei in the specimen, when the transferred energy overcomes the displacement energy of the atoms in the material, and it is dominant at high energy. ${ }^{26,28,29}$ Ionization damage (or radiolysis) involves inelastic scattering, and is dominant at low energy. ${ }^{28,29}$ In the present case, for a better comprehension of the electron-specimen interaction we evaluated the evolution of halide perovskite NCs, which involved appreciable compositional change, upon electron beam irradiation at two different values of incident electron energy $\left(E_{0}\right)$ (see Figure $S 1$ ). In particular, we verified that 80 $\mathrm{keV}$ electrons cause more effective damage (in terms of loss of $\mathrm{Br}$ atoms) than $200 \mathrm{keV}$ electrons, suggesting that radiolysis rather than knock-on is the major mechanism of damage, as expected for both covalent and ionic crystals. ${ }^{29}$ Therefore, a thorough analysis of the compositional change of the perovskite
NCs upon irradiation was carried out at $E_{0}=80 \mathrm{keV}$ from areas containing thin nanosheets $(3 \mathrm{~nm}$ thick, prepared as reported in our previous work ${ }^{14}$ ) at room temperature (RT), by acquiring energy-dispersive X-ray spectroscopy (EDS) spectra at regular time intervals up to a total accumulated dose of $6.5 \times 10^{4} \mathrm{e}^{-} /$ $\AA^{2}$. Note that the starting $3 \mathrm{~nm}$ thick nanosheets were Csdeficient $\left(\mathrm{Cs}_{1-x} \mathrm{PbBr}_{3}\right.$, Figure S1), while the thicker crystals (for example, > $10 \mathrm{~nm}$ thick nanosheets) had the expected $\mathrm{CsPbBr}_{3}$ stoichiometry. However, for the sake of simplicity, the stoichiometric formula $\left(\mathrm{CsPBr}_{3}\right)$ will be used to address also the $3 \mathrm{~nm}$ thick nanosheets throughout this work.

Figure 1(a) reports EDS spectra (in the energy range of interest) at increasing doses from scanning TEM (STEM)-EDS
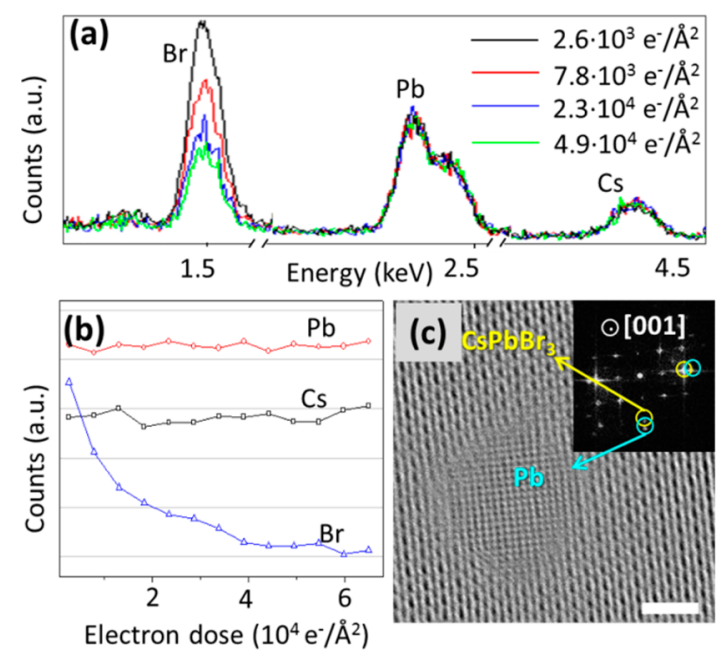

Figure 1. $\mathrm{Br}$ desorption and $\mathrm{Pb}$ nanoparticle formation during electron irradiation of $3 \mathrm{~nm}$ thick $\mathrm{CsPbBr}_{3}$ nanosheets at RT. (a) EDS spectra in the energy range of interest at increasing electron doses $\left(E_{0}=80 \mathrm{keV}\right)$; (b) Plot of the net integrated peak intensity for $\mathrm{Cs}, \mathrm{Pb}$, and $\mathrm{Br}$ as a function of the electron dose from STEMEDS analysis $\left(E_{0}=80 \mathrm{keV}\right)$; (c) HRTEM of a Pb nanoparticle formed on $\mathrm{CsPbBr}_{3}$ nanosheet, with both $\mathrm{CsPbBr}_{3}$ and $\mathrm{Pb}$ oriented along the $[001]$ zone axis (Scale bar: $2 \mathrm{~nm}, E_{0}=200 \mathrm{keV}$, dose: $\left.1140 \mathrm{e}^{-} / \AA^{2}\right)$, and (inset) Fast Fourier Transform (FFT) with the spots for $\mathrm{CsPbBr}_{3}\{220\}$ and $\mathrm{Pb}\{200\}$ circled.

analysis. Compositional changes were plotted by measuring the net peak intensity (proportional to the number of atoms) of the three elements of interest (Figure 1(b)). Upon irradiation, Cs and $\mathrm{Pb}$ intensities did not change appreciably, while the signal from $\mathrm{Br}$ decreased, especially at higher electron dose. This was also evident in the EDS elemental maps acquired at the boundary regions between areas exposed to different electron doses (see Figure S2). Note that an initial $\mathrm{Br}: \mathrm{Pb}$ ratio lower than the stoichiometric one (3:1) was most likely caused by electron induced $\mathrm{Br}$ loss as soon as the EDS measurement was started. Such loss of $\mathrm{Br}$ atoms is analogous to the electron stimulated desorption of fluorine in $\mathrm{CaF}_{2}$, or of oxygen in several oxides, as said before. ${ }^{26}$ In the case of $\mathrm{CaF}_{2}, \mathrm{~F}^{-}$is oxidized to $\mathrm{F}^{0}$ or to $\mathrm{F}^{+}$, and likewise in oxides $\mathrm{O}^{2-}$ is oxidized to $\mathrm{O}^{0}$ or to $\mathrm{O}^{+}$. The neutral species $\left(\mathrm{F}^{0}\right.$ or $\left.\mathrm{O}^{0}\right)$ diffuse and are desorbed due to their low reactivity. When positively charged species are formed, the Madelung potential is repulsive, and such species would be desorbed quickly when they are close to the surface. ${ }^{26}$ In our samples (and as already reported by others $\left.^{12-15}\right)$, in parallel with the $\mathrm{Br}$ loss, high contrast particles were formed during electron beam irradiation on $\mathrm{CsPbBr}_{3}$ 

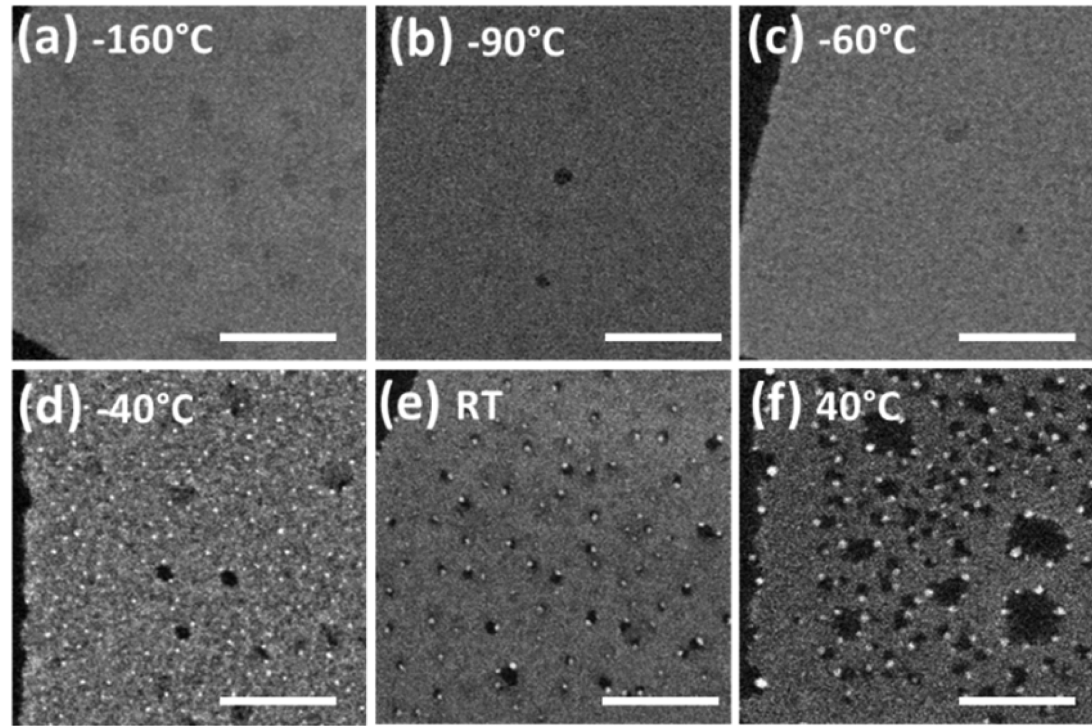

Figure 2. Effect of temperature on the nucleation and growth of $\mathrm{Pb}$ nanoparticles in $3 \mathrm{~nm}$ thick $\mathrm{CsPbBr}_{3}$ nanosheets, as shown in $\mathrm{HAADF}$ STEM images (Scale bars: $50 \mathrm{~nm}, E_{0}=200 \mathrm{keV}$, the total doses for acquiring all the images are approximately $\left.\sim 1.0 \times 10^{3} \mathrm{e}^{-} / \AA^{2}\right):(\mathrm{a})-160$ ${ }^{\circ} \mathrm{C}$; (b) $-90{ }^{\circ} \mathrm{C}$; (c) $-60{ }^{\circ} \mathrm{C}$; (d) $-40{ }^{\circ} \mathrm{C}$; (e) $\mathrm{RT}$; (f) $40{ }^{\circ} \mathrm{C}$.

(orthorhombic phase, ICSD 97851), and these were identified as metal $\mathrm{Pb}$ nanoparticles with face centered cubic structure (ICSD 96501, see high-resolution TEM (HRTEM) image in Figure 1(c)).

We can therefore state that such transformations are due to electron stimulated desorption of $\mathrm{Br}$ and reduction of $\mathrm{Pb}^{2+}$ to $\mathrm{Pb}^{0}$. From Figure 1(a), the maximum fractional loss of $\mathrm{Br}$ was close to 2/3. Assuming that all the $\mathrm{Pb}^{2+}$ ions were reduced at the maximum loss of $\mathrm{Br}$, we infer that the reduction of one $\mathrm{Pb}^{2+}$ ion was accompanied by the loss of two $\mathrm{Br}$ atoms, and this implies that a considerable fraction of $\mathrm{Br}^{-}$ions was very likely oxidized to $\mathrm{Br}^{0}$, and also that such oxidized species could diffuse and were ultimately desorbed from the NCs. A similar process was found to affect $\mathrm{CsPbX}_{3} \mathrm{NCs}$ with different halogens $(\mathrm{X}=$ $\mathrm{Cl}, \mathrm{I}$ ) and even $\mathrm{Cs}_{4} \mathrm{PbBr}_{6} \mathrm{NCs}$ (Figure S3). Details on the syntheses of these nanocrystals are found in the Supporting Information (SI). We also found that even irradiation by X-rays, by using the X-ray source of a X-ray photoelectron spectrometer (XPS), can cause a slight reduction of $\mathrm{Pb}^{2+}$ and a slight desorption of $\mathrm{Br}$ (see Figure S4). Therefore, our results and the observation of the $\mathrm{Pb}^{0}$ peak in recent XPS studies of organic lead halide perovskites ${ }^{8,30}$ imply that the $\mathrm{Br}$ desorption (and $\mathrm{Pb}^{2+}$ reduction) can be triggered by different types of radiation and can be generally classified as a stimulated desorption process. On the other hand, under irradiation by UV laser light lead halide perovskite NCs may undergo degradation via a different mechanism, as reported by Wang et $a l^{31}$

As mentioned above, the damage of $\mathrm{CsPbBr}_{3}$ nanosheets was slower at higher incident electron energy $\left(E_{0}=200 \mathrm{keV}\right)$, hence this condition was chosen to capture the details of the following stage of evolution. Our experiments clearly show that $\mathrm{Pb}^{0}$ atoms aggregated to form $\mathrm{Pb}$ nanoparticles at a rate that depended on the temperature (see high angle annular dark field (HAADF)-STEM images in Figure 2). At low temperatures (i.e., $-60{ }^{\circ} \mathrm{C}$ or lower), $\mathrm{Br}$ atoms were desorbed (Figure $\mathrm{S} 1(\mathrm{~b}, \mathrm{c})$ ) and $\mathrm{Pb}^{2+}$ ions were most likely reduced to $\mathrm{Pb}^{0}$, according to the electron-stimulated desorption mechanism described above. However, no $\mathrm{Pb}$ nanoparticles were visible (see Figure 2(a-c) and their HRTEM images in Figure S5(a)). This result suggests that, at these temperatures, $\mathrm{Pb}^{0}$ atoms did not have enough energy to overcome the activation barrier for diffusion, which would have enabled them to cluster into $\mathrm{Pb}$ particles at the surface of the NCs. Still, in this temperature range one cannot exclude that clusters containing only a few atoms ( $\mathrm{Pb}$ nanoclusters) were formed, but in that case they were simply not discernible in the images. At higher temperatures (i.e., $-40{ }^{\circ} \mathrm{C}$ or above), and still under irradiation, $\mathrm{Pb}^{0}$ atoms (and probably also clusters of $\mathrm{Pb}$ atoms) could instead diffuse and coalesce into observable $\mathrm{Pb}$ nanoparticles $^{23,32}$ (Figure 2(d-f) and Figure S5(b)). As a general trend, higher temperatures promoted the formation of larger $\mathrm{Pb}$ particles (compare for example Figures $2 \mathrm{~d}-\mathrm{f}$ ).

A question arises on whether electron beam heating has an important effect here. This is certainly true in materials with poor thermal conductivity. The two parameters that play a pivotal role in electron beam heating are dose rate (proportional to the beam current) and incident electron energy $\left(E_{0}\right)$. In the high temperature conditions at which visible $\mathrm{Pb}$ nanoparticles are formed, a study on the dependence of $\mathrm{Pb}$ nanoparticle formation on the irradiation dose rate for $200 \mathrm{keV}$ electrons is reported in Figure S6. It shows that, in the range between $8.5 \times 10^{5}$ and $2.0 \times 10^{8} \mathrm{e}^{-} / \AA^{2} / \mathrm{s}$, the increase of size of $\mathrm{Pb}$ nanoparticle with increasing dose rate is negligible. The dose rate used in typical HRTEM and STEM imaging is within this range. Nevertheless, a higher dose rate induces the production of larger $\mathrm{Pb}$ nanoparticles, as demonstrated in Figure S6. We also found that at low temperatures $\left(-60{ }^{\circ} \mathrm{C}\right.$ or lower, which were conditions that did not promote the formation of observable $\mathrm{Pb}$ nanoparticles), with the same dose rate $\left(5.0 \times 10^{6} \mathrm{e}^{-} / \AA^{2} / \mathrm{s}\right)$ used in Figure $2(\mathrm{a}-\mathrm{c})$ not even electron irradiation with ten times higher doses could induce the appearance of $\mathrm{Pb}$ nanoparticles. This suggests that a significant temperature rise does not occur even under irradiation with much higher doses. These observations evidence that electron beam heating of $\mathrm{CsPbBr}_{3}$ has a negligible effect at our experimental conditions for $E_{0}=200 \mathrm{keV} .80 \mathrm{keV}$ electrons would have a thermal effect that is approximately 1.6 


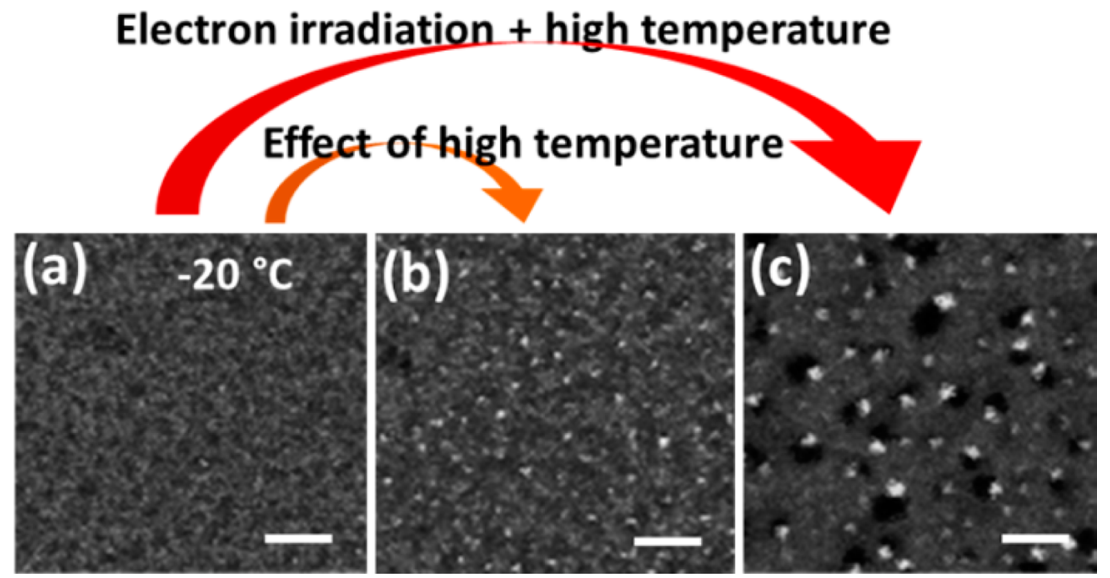

Figure 3. HAADF-STEM images of $3 \mathrm{~nm}$ thick nanosheets (Scale bars: $20 \mathrm{~nm}, E_{0}=200 \mathrm{keV}$ ): (a) Initially at $-20^{\circ} \mathrm{C}$; (b) Following the acquisition of the image (a), the substrate temperature was raised to RT for 10 min with electron beam blanked, and again cooled to $-20{ }^{\circ} \mathrm{C}$ for imaging of the same nanosheet; (c) image acquired at RT.

times the one from $200 \mathrm{keV}$ electrons, which is still negligible within the dose rate employed in this work (see a more detailed discussion in the SI). In two different irradiation experiments, carried out at the higher temperature side of our tested interval $\left(-40{ }^{\circ} \mathrm{C}\right.$ and RT, see Figure $\left.2(\mathrm{~d}, \mathrm{e})\right)$, we counted the numbers of $\mathrm{Pb}^{0}$ atoms within the observed nanoparticles and found them comparable $\left(\sim 5 \times 10^{5}\right.$ in total, the Methods section explains how the calculations were made). This suggests that, although the perovskite lattice collapses locally, the $\mathrm{Pb}^{0}$ species are not lost from the NCs, a result that goes hand in hand with the quantitative EDS results reported in Figure 1. Instead, they are mainly sequestered in the formation of the metallic clusters, if the temperature is high enough.

Both temperature and electron irradiation have a role in the nucleation and growth of $\mathrm{Pb}^{0}$ clusters. The effect of temperature has already been mentioned, and was made more evident by the control experiments reported in Figure 3. In this case, the sample was first irradiated at $-20{ }^{\circ} \mathrm{C}$, after which the temperature was raised (to RT). If, during this latter step, the electron beam was blanked (i.e., it was prevented to hit the sample), the initial, barely visible $\mathrm{Pb}$ nanoparticles that had formed at $-20{ }^{\circ} \mathrm{C}$ evolved into larger particles (diameter $\leq 1.8$ $\mathrm{nm}$, an average value is hard to evaluate due to their small sizes), supporting the idea of temperature promoted diffusion of $\mathrm{Pb}^{0}$ species. If the temperature was raised while the sample was being irradiated, considerably larger $\mathrm{Pb}$ nanoparticles $(2.3$ $\pm 0.8 \mathrm{~nm}$ ) were formed (see Figure 3 ). The electron induced heating is negligible at the used dose rate $8.5 \times 10^{5} \mathrm{e}^{-} / \AA^{2} / \mathrm{s}$ as discussed above and in Figure S6. It is likely that this occurred as the electron irradiation contributes to enhancing the diffusivity of $\mathrm{Pb}^{0}$ species and of small $\mathrm{Pb}$ nanoparticles by transferring momentum and energy to them. ${ }^{23,33}$ In addition, new $\mathrm{Pb}^{0}$ species were continuously generated by irradiation. This implies that, although the initial $\mathrm{Br}$ desorption and $\mathrm{Pb}^{2+}$ reduction is mainly due to a radiolysis process, we should also consider a contribution of knock-on displacement in the $\mathrm{Pb}$ nanoparticle nucleation process.

In the high temperature range, the $\mathrm{Pb}^{0}$ atoms can form the $\mathrm{Pb}$ nanoparticles by diffusing both along the surface and through the inner regions of the perovskite NCs, although diffusion from the bulk should be less marked in thicker samples. We compared the behavior of thin nanosheets $(3 \mathrm{~nm}$ thick) with that of the thicker sheets (tens of nm thick) that were also present in the sample (note that the thicker sheets represented only a minor fraction of the sample). The comparison was quantified through compositional change under the same irradiation conditions. As shown in Figure S7, the Br concentration (at.\%) dropped from $57 \%$ to $50 \%$ for $3 \mathrm{~nm}$ thick nanosheets, while it remained nearly constant at $59 \%$ for thicker nanosheets. The comparison evidenced that the thick nanosheets had undergone much less change and consequently less damage compared to the thin nanosheets (see Figure S7). This implies that both desorption of $\mathrm{Br}$ and aggregation of $\mathrm{Pb}^{0}$ species occur preferentially at the surface, which is expected since the surface is generally characterized by a lower barrier to atomic diffusion. Also, both $\mathrm{Br}$ and $\mathrm{Pb}$ atoms have lower coordination at the surface and their electronic configuration may be more easily altered by electron irradiation and additionally $\mathrm{Br}$ species are more easily desorbed when they are bound to surface sites than to inner regions of the sheets. As a consequence, the surface is energetically less stable than the bulk and plays an important role in the electron beam-induced degradation of perovskite NCs. Similar conclusions were drawn by Philippe et al. ${ }^{8}$ and Yuan et al. ${ }^{9}$ by exposing $\mathrm{APbX}_{3}$ thin films to different irradiations.

In addition to nanosheets, we also tested colloidal $\mathrm{CsPbBr}$ NCs with nanocuboid and nanowire shapes and different sizes, for which the syntheses are found in the Methods section. Overall, we found that the samples with higher surface-tovolume ratio ( $3 \mathrm{~nm}$ thick nanosheets, nanowires with diameters smaller than $10 \mathrm{~nm}$, and nanocuboids with $8 \mathrm{~nm}$ edge) were more susceptible to electron irradiation than the samples with lower surface-to-volume ratio (see Figure 4, S7 and S8). The latter included: nanosheets that were tens of $\mathrm{nm}$ thick, nanowires wider than $10 \mathrm{~nm}$, and cuboids with 20-40 nm edge (see Figures 4 and S7). In these larger NCs, the internal structure appeared to be preserved under irradiation, and only a few $\mathrm{Pb}$ nanoparticles were formed at their surface. On the other hand, lowering the incident electron energy to $80 \mathrm{keV}$ could cause significant damage even to these NCs, due to the increased probability of inelastic interaction. An increase in temperature could also promote the process by speeding up the diffusion along the surface, as well as by promoting the diffusion from the inner regions of the NCs (above $60{ }^{\circ} \mathrm{C}$ ), and this led to their fast degradation (see Figure S9). The dynamic evolution at high temperature for large nanocuboids is 


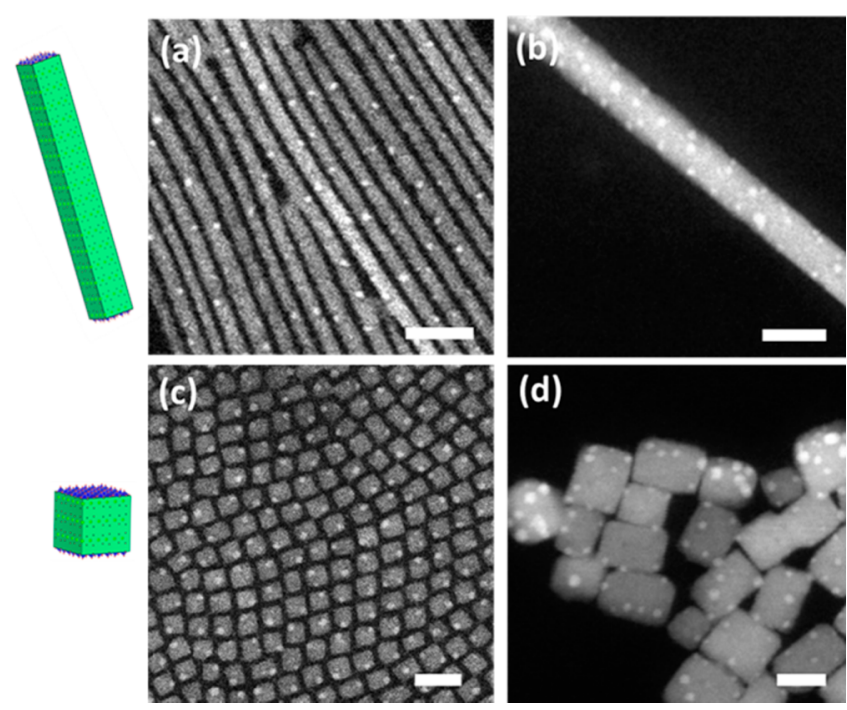

Figure 4. HAADF-STEM images showing preferential formation of $\mathrm{Pb}$ nanoparticles at the edges and corners of $\mathrm{CsPbBr}_{3} \mathrm{NCs}$ : (a) 5 nm wide nanowires; (b) $20 \mathrm{~nm}$ wide nanowire; (c) nanocuboids with $8 \mathrm{~nm}$ edge; (d) nanocuboids with 20-40 $\mathrm{nm}$ edge (Scale bars: $\left.20 \mathrm{~nm}, E_{0}=200 \mathrm{keV}, \mathrm{RT}\right)$

demonstrated in the Supporting Video S1. High surface-tovolume ratio NCs readily decomposed, which is clearly demonstrated in Figure 4. The $5 \mathrm{~nm}$ wide nanowires broke due to the formation of $\mathrm{Pb}$ nanoparticles. It is also remarkable that these $\mathrm{Pb}$ nanoparticles were rather regularly spaced along the wires. The nanocuboids with edge length of $8 \mathrm{~nm}$ decomposed by forming $\mathrm{Pb}$ nanoparticles at the corners (see Figure S8). A close investigation of Figure 4 revealed that edges and corners of the NCs are preferential sites for $\mathrm{Pb}$ particle formation.

The starting sites of $\mathrm{Pb}$ reduction and $\mathrm{Br}$ desorption may be linked to defects. At a defect site, $\mathrm{Pb}$ ions may undergo reduction more easily. Starting preferentially from the defects, on $3 \mathrm{~nm}$ thick nanosheet voids with $\mathrm{Pb}$ nanoparticles on the edge are created. The formation of these $\mathrm{Pb}$ nanoparticles consumes part of the $\mathrm{Pb}$ from the surrounding perovskite lattice and leads to the formation of few small $\mathrm{Pb}$ nanoparticles at the surrounding locations. Overall, these processes result in the inhomogeneous damage in a single nanosheet (as can be seen in Figure 2 and Figure 3), even though the whole nanosheet is under homogeneous electron irradiation.

$\mathrm{To}$ monitor the formation of $\mathrm{Pb}$ nanoparticles at different sites under identical irradiation conditions, we took advantage of the large field of view of the direct electron detection camera combined with the large lateral size of the nanosheet to record them in a single HRTEM image (Figure 5(a)). Magnified views from the same image are displayed in Figure $5(\mathrm{~b}-\mathrm{f})$; i.e., all the events in these regions occurred simultaneously under the same irradiation conditions, which show as an eye-catching feature that the evolution of $\mathrm{Pb}$ nanoparticles at different sites is not synchronous. Some of the $\mathrm{Pb}$ nanoparticles exhibited a singlecrystal structure, anisotropic shape (i.e., square/hexagonal projection) and well-defined orientation relationship (epitaxy) with the parent $\mathrm{Cs} \mathrm{PbBr}_{3}$ nanosheet (orthorhombic phase with ICSD: 97851, [001]-oriented on the support film). The relationships were: $\mathrm{CsPbBr}_{3}$ [001] $\| \mathrm{Pb}[001], \mathrm{CsPbBr}_{3}$ (110) $\| \mathrm{Pb}$ (100), as shown for the particles in Figure 5(b,c) and for some of the particles of Figure 5(d,e). This suggests
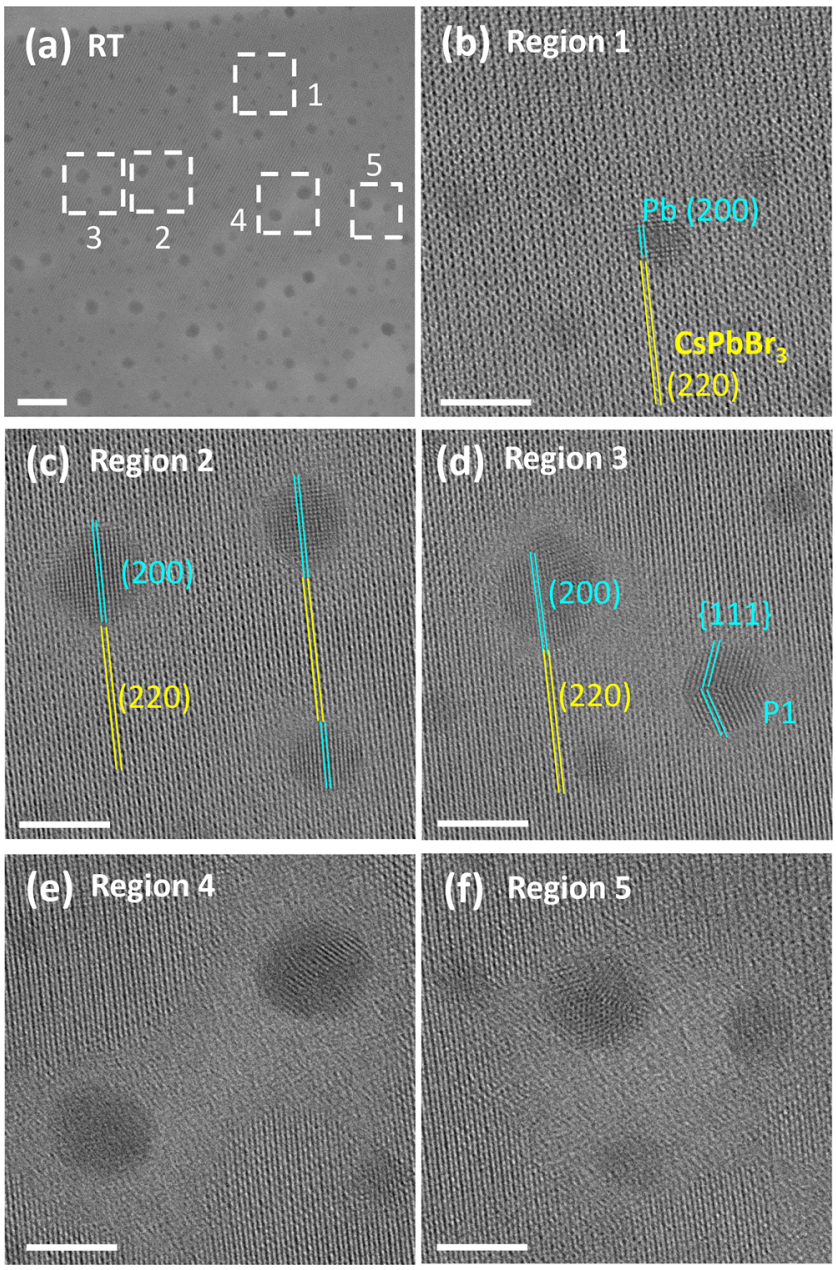

Figure 5. HRTEM analysis of $\mathrm{Pb}$ nanoparticle formation on a $3 \mathrm{~nm}$ thick $\mathrm{CsPbBr}_{3}$ nanosheet at RT: (a) Overview (Scale bar: $20 \mathrm{~nm}, E_{0}$ = $200 \mathrm{keV}$, total dose: $\left.1140 \mathrm{e}^{-} / \AA^{2}\right) ;(\mathrm{b}-\mathrm{f})$ Magnified view of five regions of interest in (a) (Scale bars: $5 \mathrm{~nm}$ ).

that the $\mathrm{Pb}$ atoms at the edge of these $\mathrm{Pb}$ nanoparticles may be bound to the parent $\mathrm{Cs} \mathrm{PbBr}_{3}$ lattice, and these are presumably early stage particles. Nonepitaxially bound, roughly spherical shapes were also found, such as the particles in Figure 5(e,f), including twinned NCs (particle P1). These nanoparticles may have resulted from a rearrangement of early stage $\mathrm{Pb}$ nanoparticles upon further irradiation. Our results support the idea that the epitaxial $\mathrm{Pb}$ particles bound to the surrounding parent lattice can survive until a critical size (i.e., number of $\mathrm{Pb}$ atoms). Above that size, the $\mathrm{Pb}$ atoms in the particle lose the epitaxial relationship with the surround lattice and rearrange, forming more isotropic particles which are thermodynamically more stable. This can be rationalized as due to two major reasons: (i) edge atoms are weakly bound to the surrounding lattice and the unstable bonds between the edge atoms and the surrounding lattice are broken due to the ongoing irradiation; and (ii) rearrangement results in the emergence of facets of lower energy. Among these more isotropic particles, some $\mathrm{Pb}$ nanocrystals exhibit twinning, a type of defect that is often seen in the case of metal nano- and microparticles, due to its low formation energy in metals. ${ }^{34}$ By feeding the $\mathrm{Pb}$ atoms to the surrounding $\mathrm{Pb}$ nanoparticles, some areas on the $\mathrm{Cs} \mathrm{PbBr}_{3}$ nanosheet turned into voids decorated by $\mathrm{Pb}$ nanoparticles at their edges (see Figure 5(e,f) and Figure 2(e,f)), while the Cs 
atoms as well as the remaining $\mathrm{Pb}$ and $\mathrm{Br}$ species were randomly distributed in the voids.

Once formed, the $\mathrm{Pb}$ nanoparticles were not stable under further electron irradiation. The evolution of the number and size distribution of the $\mathrm{Pb}$ nanoparticles is reported in Figure 6 .

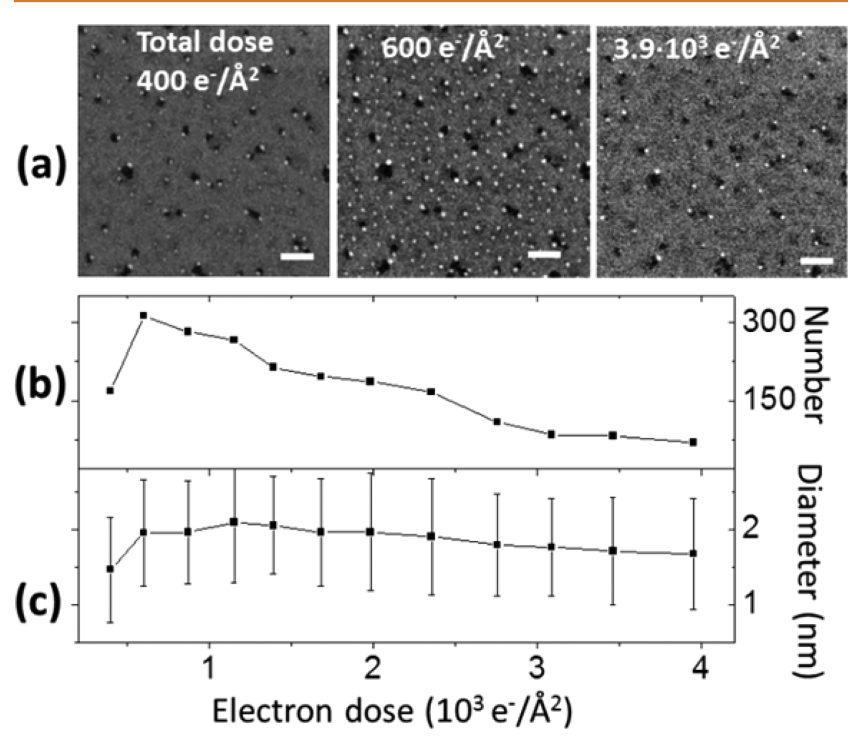

Figure 6. Evolution of number and size of $\mathrm{Pb}$ nanoparticles on 3 $\mathrm{nm}$ thick $\mathrm{CsPbBr}_{3}$ nanosheets at $\mathrm{RT}$ as a function of electron irradiation $\left(E_{0}=200 \mathrm{keV}, \mathrm{RT}\right)$. (a) HAADF-STEM images at four doses (Scale bars: $20 \mathrm{~nm}$ ); (b) Number of $\mathrm{Pb}$ nanoparticles versus electron dose; (c) Average size of $\mathrm{Pb}$ nanoparticles versus electron dose (the diameter is calculated from the area by assuming a circular shape, error bar is the standard deviation).

At an electron dose of $400 \mathrm{e}^{-} / \AA^{2}$, around $170 \mathrm{~Pb}$ nanoparticles had nucleated in the area shown in the HAADF-STEM image in Figure 6(a). This number rapidly increased to 310 at a dose of $600 \mathrm{e}^{-} / \AA^{2}$ (Figure $6(\mathrm{a}, \mathrm{b})$ ). These nanoparticles were not stable and were dismantled under further electron irradiation: the number decreased to 70 at a dose of $3.9 \times 10^{3} \mathrm{e}^{-} / \AA^{2}$ (Figure 6(a,b)), and the particles basically disappeared at even higher doses. The same holds for $\mathrm{Pb}$ nanoparticles produced at different temperatures (see Figure S10). The size distribution of the $\mathrm{Pb}$ nanoparticles also changed during electron irradiation. For the $\mathrm{Pb}$ nanoparticles formed in Figure 6(a), the average size was $1.5 \mathrm{~nm}$ at a dose of $400 \mathrm{e}^{-} / \AA^{2}$ and increased to $2.1 \mathrm{~nm}$ at a dose of $1.1 \times 10^{3} \mathrm{e}^{-} / \AA^{2}$. At a given temperature (i.e., surface diffusion rate), an equilibrium size was reached and did not increase any longer, and instead started to gradually decrease to $1.7 \mathrm{~nm}$ at a dose of $3.9 \times 10^{3} \mathrm{e}^{-} / \AA^{2}$.

The dominant reason for $\mathrm{Pb}$ particle size variation with increasing total dose is not heating. Note that, the slow variation of the average diameter of the $\mathrm{Pb}$ nanoparticles over time was due to the asynchronous evolution they underwent at different sites. According to our analysis based on the elemental maps of Figure $\mathrm{S} 11, \mathrm{a} \mathrm{Pb}$ nanoparticle forms and then its size decreases under further electron irradiation, until the particle finally disappears. As the $\mathrm{Pb}$ composition recorded in the analyzed areas is constant (see the discussion for Figure 1) the dissolution of the $\mathrm{Pb}$ nanoparticle is unlikely to be the result of a sublimation or vaporization process. We also tend to exclude the participation of metallic $\mathrm{Pb}$ atoms in redox reactions under electron irradiation conditions, even though the surroundings of the $\mathrm{Pb}$ particles are chemically active. This is because, to do so, the metallic $\mathrm{Pb}$ atoms would have to stably lose electrons (i.e., be oxidized), which is unlikely when electrons are continuously supplied by irradiation.

The mechanism of dissolution of the $\mathrm{Pb}$ nanoparticles is revealed by the HRTEM study in Figure S12-S14. Following the amorphization of the $\mathrm{Pb}$ nanoparticles (Figure S12 and Figure S13), with further irradiation, $\mathrm{Pb}$ atoms spread out on the supporting film. In the case of $3 \mathrm{~nm}$ thick nanosheet, on which $\mathrm{Pb}$ nanoparticles decorate the edge of voids, the dissolution of $\mathrm{Pb}$ nanoparticles under further electron irradiation causes the decrease of the size of the voids, as observed in Figure 6. The dissolution of $\mathrm{Pb}$ nanoparticles from one isolated nanocuboid leads to continuous decomposition of the nanocuboid (Figure S13), while for an array of closely spaced nanocuboids the spread atoms accumulate at the regions between cuboids and appear as hollow frames that follow the initial contours of the cuboids (Figure S14). The atoms that have diffused from adjacent $\mathrm{Pb}$ nanoparticles could gather and form clusters (see Figure S15). This process is similar to the

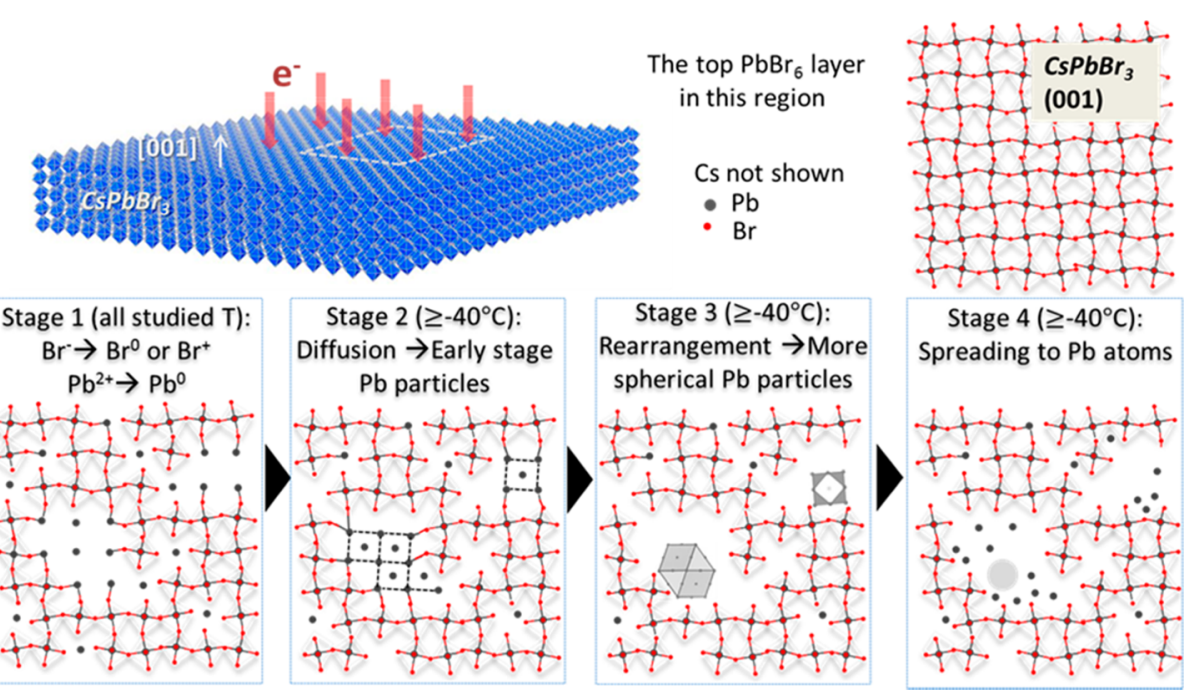

Figure 7. Schematics depicting the stages in the evolution of an irradiated area in a $\mathrm{CsPbBr}_{3}$ nanosheet ([001] orientation). Only the atomic model for the topmost layer of $\mathrm{PbBr}_{6}$ octahedra is shown for clarity. 
reported dissipation of $\mathrm{Pd}$ and Mo nanoparticles through diffusion under electron beam irradiation. ${ }^{35}$ Therefore, if we rule out the possibility of vaporization and redox reactions, the most probable mechanism for the "disappearance" of $\mathrm{Pb}$ nanoparticles under prolonged electron irradiation is through diffusion and spreading out of $\mathrm{Pb}$ atoms on the remaining perovskite lattice and on the carbon film of the TEM grid.

To summarize the above-presented results, the overall process of electron beam-induced transformations in $\mathrm{Cs} \mathrm{PbBr}_{3}$ NCs is schematically summarized in Figure 7. For demonstration purposes, we show the evolution of $\mathrm{Pb}$ nanoparticles in an irradiated area, and we draw only the topmost $\mathrm{PbBr}_{6}$ octahedron layer. Considering that, in the $\mathrm{CsPbBr}_{3}$ structure, mainly covalent bonds form between $\mathrm{Pb}$ and $\mathrm{Br}$, and mainly ionic bonds form between $\mathrm{Cs}$ and $\mathrm{Br}$, while $\mathrm{Pb}$ and $\mathrm{Cs}$ atoms are not bound to each other, ${ }^{36}$ the following stages can be identified on the basis of the above results:

Stage 1. The whole process starts with the oxidation of $\mathrm{Br}^{-}$ and reduction of $\mathrm{Pb}^{2+}$ at all studied temperatures (Figures 1 and Figure S1), which is likely to occur preferentially at the corners and edges of the surface of the NC. Oxidized halide species desorb from the surface.

Stage 2. At high temperatures (above $-40{ }^{\circ} \mathrm{C}$ ), in tandem with electron induced motion (i.e., by momentum transfer), the reduced $\mathrm{Pb}^{0}$ atoms diffuse preferentially along the surface and aggregate into single-crystal $\mathrm{Pb}$ nanoparticles, which possess anisotropic shapes and epitaxial relationships with the parent nanosheet lattice, probably due to partial binding to the surrounding lattice. Note that a higher temperature (above 60 ${ }^{\circ} \mathrm{C}$ ) can enable the diffusion of $\mathrm{Pb}^{0}$ also from the inner perovskite lattice.

Stage 3. The bonds between the early $\mathrm{Pb}$ nanoparticles and the parent $\mathrm{Cs} \mathrm{PbBr}_{3} \mathrm{NCs}$ are broken upon further irradiation, hence the $\mathrm{Pb}$ nanoparticles evolve toward more isotropic shapes, giving rise to voids. The $\mathrm{Pb}$ particles are located at the boundary between the voids and the remaining perovskite lattice (as shown in Figures 2 and 6).

Stage 4. Upon further electron irradiation, the $\mathrm{Pb}$ nanoparticles amorphize, then they become smaller and finally fade as the $\mathrm{Pb}$ atoms spread on the remains of the $\mathrm{Cs} \mathrm{PbBr}_{3}$ surface or on the support film.

\section{CONCLUSIONS}

The experiments presented here demonstrate that the electron beam induced nucleation of $\mathrm{Pb}$ nanoparticles on $\mathrm{CsPbX}_{3} \mathrm{NCs}$ is mainly due to a radiolysis damage process. Electron irradiation $(80 \mathrm{keV} / 200 \mathrm{keV})$ induces the desorption of halogen atoms from the surface of the NCs and the reduction of $\mathrm{Pb}^{2+}$ to metallic $\mathrm{Pb}^{0}$, in analogy with the electron stimulated desorption mechanism reported in the literature. This process also occurs under other types of irradiation, such as X-rays. Subsequently, adjacent $\mathrm{Pb}^{0}$ atoms diffuse and aggregate into $\mathrm{Pb}$ nanoparticles at a temperature dependent rate, which is simultaneously aided by electron irradiation, most probably due to electron beam-induced motion via knock-on displacement of the $\mathrm{Pb}^{0}$ atoms on the surface. At temperatures high enough for diffusion to occur, on $\mathrm{CsPBBr}_{3} \mathrm{NCs}$, anisotropic $\mathrm{Pb}$ nanoparticles epitaxially oriented and bound to the parent structure are produced. These rearrange into more spherical particles under further irradiation, after the bonds between their edge atoms and the surrounding parent $\mathrm{CsPbBr}_{3} \mathrm{NCs}$ are broken. The nucleated $\mathrm{Pb}$ nanoparticles are unstable and are dismantled under further electron irradiation. The formation of
$\mathrm{Pb}$ nanoparticles preferentially occurs at the corners and edges of the surface of the NCs. In this context, $\mathrm{CsPbBr}_{3} \mathrm{NCs}$ with higher surface-to-volume ratio are prone to decompose by electron beam-induced $\mathrm{Pb}$ nanoparticles formation, while lower surface-to-volume ratio $\mathrm{CsPbBr}_{3} \mathrm{NCs}$ are more robust. We could also safely exclude any significant electron beam heating under our experimental conditions.

The mechanism presented in this study may also govern the formation of high contrast particles observed in TEM images of hybrid lead halide perovskite $\mathrm{NCs}^{37}$ tin halide perovskite $\mathrm{NCs}^{38}$ and other perovskite related materials such as $\mathrm{CsPb}_{2} \mathrm{Br}_{5}$, ${ }^{39}$ and it helps defining some precautions to follow. When dealing with halide perovskites characterization using electron microscopy, increasing the incident electron energy of the microscope can largely improve the stability of the sample. By lowering the substrate temperature, the characterization of high aspect ratio perovskite NCs becomes feasible as a consequence of the retarded decomposition process. Besides, radiation induced damage may be exploited for patterning purposes, i.e., by creating $\mathrm{Br}$-rich and $\mathrm{Br}$-deficient areas, or for selectively inducing anion exchange in different regions, to enable patterned luminescent surfaces, where each pixel in the display device should correspond to the whole irradiated area, in order to avoid the inhomogeneity caused by the electron beam. Besides, in principle the $\mathrm{Pb}$ formation process can be used to make porous perovskite NCs. These can be advantageous compared to nonporous perovskite NCs in sensing applications, because the analytes can penetrate into the pores and interact more strongly with the perovskite. Another potential application is to enable a cation exchange process with the aid of the $\mathrm{Pb}^{2+}$ reduction under electron irradiation to produce a shell of perovskite with another element on the surface, as a route toward fabricating core-shell perovskite NCs.

\section{METHODS}

Electron Beam Irradiation in the TEM. The electron beam irradiation experiments were carried out in a $200 \mathrm{kV}$ microscope (JEOL JEM-2200FS) equipped with a spherical aberration corrector (CEOS) for the objective lens and an in-column image filter ( $\Omega$-type). The acceleration voltage can be lowered to $80 \mathrm{kV}$ by means of an electric short switch. The base pressure in the TEM column was $2 \times$ $10^{-7}$ mbar.

High Resolution TEM (HRTEM). The electron dose is reported for each HRTEM image. The images were acquired with two types of detectors: a CCD camera with $2048 \times 2048$ pixels (UltraScan 1000, Gatan Inc.), and a direct electron detection camera (K2 Summit, Gatan Inc.), with $7420 \times 7676$ pixels in super-resolution mode. The $\mathrm{K} 2$ camera is able to capture images on a large field of view with atomic resolution, while minimizing electron exposure. The HRTEM images were acquired with the $\mathrm{K} 2$ camera unless specified.

Probe Current Measurements. We first calibrated the electron counts on the CCD camera using the direct electron detection camera for which electron number is directly counted. Then the probe current was measured by acquiring the Scanning TEM (STEM) probe on UltraScan 1000. With a spot size of $1.5 \mathrm{~nm}$ and convergence semiangle of $16 \mathrm{mrad}$, the resulting probe current was $68 \mathrm{pA}$ (corresponding to a current density of $3.8 \times 10^{3} \mathrm{~A} / \mathrm{cm}^{2}$ ) and was varied by selecting the spot size.

High Angle Annular Dark Field (HAADF-STEM) and Energy Dispersive X-ray Spectrometry (EDS) Analysis. A Bruker Quantax 400 EDS system with an XFlash 5060 detector was used for elemental analysis. The NCs composition was estimated from the EDS spectra in the thin film approximation (Cliff-Lorimer) by fitting the $\mathrm{Cs} \mathrm{L} \alpha, \mathrm{Pb}$ $\mathrm{L} \alpha$ and $\mathrm{Br} \mathrm{K} \alpha$ lines, with a relative error of $10 \%$ for $\mathrm{Cs}, 10 \%$ for $\mathrm{Pb}, 4 \%$ for $\mathrm{Br}$, evaluated as the standard deviation in the fit. 
Temperature Dependent Study. A JEOL EM-21130 specimen heating holder with an electric furnace and EM-SHU2 heater control was used for observing the effect of higher temperatures on electron beam-induced transformations in $\mathrm{CsPbBr}_{3}$ nanocrystals. A Gatan cryotransfer holder with liquid nitrogen cooling equipped with a Model 900 Smartset cold stage controller was used for observing the electron beam-induced transformation at low temperatures.

X-ray Photoelectron Spectroscopy (XPS). A Kratos Axis Ultra DLD spectrometer equipped with a monochromatic $\mathrm{Al} \mathrm{K \alpha}$ source (photon energy $=1486.6 \mathrm{eV}$ ) was used for irradiation and characterization. Nanosheets were exposed to continuous X-ray exposure at a photon flux of $2.4 \times 10^{11}$ photons $/ \mathrm{mm}^{2} \mathrm{~s}$. Highresolution spectra were acquired with an analyzer pass energy of $10 \mathrm{eV}$ and steps of $0.1 \mathrm{eV}$. The pressure in the chamber was kept below $10^{-8}$ mbar and the takeoff angle set at $\Phi=0^{\circ}$. Energy calibration was performed fixing the $\mathrm{C}-\mathrm{C}$ component of $\mathrm{C} 1 \mathrm{~s}$ spectrum at $284.8 \mathrm{eV}$. Relative atomic percentages of different species were computed from high-resolution spectra, fitted with Voigt functions using CasaXPS software.

Synthesis of $\mathrm{CsPbBr}_{3}$ Nanocrystals. Thin, rectangular shaped nanosheets around $3 \mathrm{~nm}$ thick, with lateral size of several $100 \mathrm{~nm}$ up to few $\mu \mathrm{m}$ were synthesized by a colloidal procedure reported elsewhere. ${ }^{14} \mathrm{CsPbBr}_{3}$ nanowires with rectangular cross section were synthesized by a colloidal procedure previously reported by us. ${ }^{15}$ $\mathrm{CsPbBr}_{3}$ nanocuboids with $8 \mathrm{~nm}$ edge were synthesized by a colloidal procedure similar to the one reported previously. ${ }^{6}$ The $\mathrm{CsPbBr}_{3}$ nanocuboids with edge length of $20-40 \mathrm{~nm}$ were synthesized following the same colloidal procedure as for $\mathrm{CsPBrr}_{3}$ nanoplatelets, except that ethanol was used in the place of acetone. ${ }^{13}$ The TEM samples were prepared by drop-casting $10 \mu \mathrm{L}$ of the colloidal suspensions onto ultrathin carbon/holey carbon coated $\mathrm{Cu}$ grids.

Calculation of $\mathrm{Pb}^{0}$ Atoms in the $\mathrm{Pb}$ Nanoparticles. The total number of $\mathrm{Pb}^{0}$ atoms contained in $\mathrm{Pb}$ nanoparticles was estimated as follows: the total area of the $\mathrm{Pb}$ nanoparticles in the HAADF-STEM images was measured in Image $\mathrm{J}^{40}$ and then the total volume of $\mathrm{Pb}$ nanoparticles was calculated and multiplied by the atomic density of crystalline $\mathrm{Pb}$ to give the total number of $\mathrm{Pb}^{0}$ atoms.

\section{ASSOCIATED CONTENT}

\section{S Supporting Information}

The Supporting Information is available free of charge on the ACS Publications website at DOI: 10.1021/acsnano.6b08324.

EDS analysis of $\mathrm{CsPbBr}_{3}$ nanosheets, $\mathrm{Pb}$ nanoparticles formation on $\mathrm{CsPbCl}_{3}$ and $\mathrm{CsPbI}_{3}, \mathrm{~Pb}^{2+}$ reduction under $\mathrm{X}$-ray exposure, effect of temperature, electron beam heating, formation of $\mathrm{Pb}$ nanoparticles on $\mathrm{CsPbBr}_{3} \mathrm{NCs}$ with different surface-to-volume ratios, instability of $\mathrm{Pb}$ nanoparticles with electron irradiation, syntheses of $\mathrm{CsPbX}_{3}(\mathrm{X}=\mathrm{Cl}, \mathrm{I})$ and $\mathrm{Cs}_{4} \mathrm{PbBr}_{6} \mathrm{NCs}(\mathrm{PDF})$

Video $\mathrm{S} 1$ : The dynamic evolution at high temperature for large $\mathrm{CsPbBr}_{3}$ nanocuboids (AVI)

\section{AUTHOR INFORMATION}

\section{Corresponding Authors}

*E-mail: rosaria.brescia@iit.it.

*E-mail: liberato.manna@iit.it.

\section{ORCID ${ }^{\circ}$}

Giovanni Bertoni: 0000-0001-6424-9102

Liberato Manna: 0000-0003-4386-7985

\section{Notes}

The authors declare no competing financial interest.

\section{ACKNOWLEDGMENTS}

The authors acknowledge funding from the European Union under grant agreement no. 614897 (ERC Grant TRANSNANO).

\section{REFERENCES}

(1) Stoumpos, C. C.; Kanatzidis, M. G. The Renaissance of Halide Perovskites and Their Evolution as Emerging Semiconductors. Acc. Chem. Res. 2015, 48, 2791-2802.

(2) Yang, W. S.; Noh, J. H.; Jeon, N. J.; Kim, Y. C.; Ryu, S.; Seo, J.; Seok, S. I. High-Performance Photovoltaic Perovskite Layers Fabricated through Intramolecular Exchange. Science 2015, 348, 1234.

(3) Song, J.; Li, J.; Li, X.; Xu, L.; Dong, Y.; Zeng, H. Quantum Dot Light-Emitting Diodes Based on Inorganic Perovskite Cesium Lead Halides $\left(\mathrm{CsPbX}_{3}\right)$. Adv. Mater. 2015, 27, 7162-7167.

(4) Zhu, H.; Fu, Y.; Meng, F.; Wu, X.; Gong, Z.; Ding, Q.; Gustafsson, M. V.; Trinh, M. T.; Jin, S.; Zhu, X. Y. Lead Halide Perovskite Nanowire Lasers with Low Lasing Thresholds and High Quality Factors. Nat. Mater. 2015, 14, 636-642.

(5) Stoumpos, C. C.; Malliakas, C. D.; Peters, J. A.; Liu, Z.; Sebastian, M.; Im, J.; Chasapis, T. C.; Wibowo, A. C.; Chung, D. Y.; Freeman, A. J.; Wessels, B. W.; Kanatzidis, M. G. Crystal Growth of the Perovskite Semiconductor $\mathrm{Cs} \mathrm{PbBr}_{3}$ : A New Material for High-Energy Radiation Detection. Cryst. Growth Des. 2013, 13, 2722-2727.

(6) Protesescu, L.; Yakunin, S.; Bodnarchuk, M. I.; Krieg, F.; Caputo, R; Hendon, C. H.; Yang, R. X.; Walsh, A.; Kovalenko, M. V. Nanocrystals of Cesium Lead Halide Perovskites $\left(\mathrm{CsPbX}_{3}, \mathrm{X}=\mathrm{Cl}, \mathrm{Br}\right.$, and I): Novel Optoelectronic Materials Showing Bright Emission with Wide Color Gamut. Nano Lett. 2015, 15, 3692-3696.

(7) Huang, H.; Polavarapu, L.; Sichert, J. A.; Susha, A. S.; Urban, A. S.; Rogach, A. L. Colloidal Lead Halide Perovskite Nanocrystals: Synthesis, Optical Properties and Applications. NPG Asia Mater. 2016, 8, e328.

(8) Philippe, B.; Park, B.-W.; Lindblad, R.; Oscarsson, J.; Ahmadi, S.; Johansson, E. M. J.; Rensmo, H. Chemical and Electronic Structure Characterization of Lead Halide Perovskites and Stability Behavior under Different Exposures-a Photoelectron Spectroscopy Investigation. Chem. Mater. 2015, 27, 1720-1731.

(9) Yuan, H.; Debroye, E.; Janssen, K.; Naiki, H.; Steuwe, C.; Lu, G.; Moris, M.; Orgiu, E.; Uji-i, H.; De Schryver, F.; Samorì, P.; Hofkens, J.; Roeffaers, M. Degradation of Methylammonium Lead Iodide Perovskite Structures through Light and Electron Beam Driven Ion Migration. J. Phys. Chem. Lett. 2016, 7, 561-566.

(10) Zhu, F.; Men, L.; Guo, Y.; Zhu, Q.; Bhattacharjee, U.; Goodwin, P. M.; Petrich, J. W.; Smith, E. A.; Vela, J. Shape Evolution and Single Particle Luminescence of Organometal Halide Perovskite Nanocrystals. ACS Nano 2015, 9, 2948-2959.

(11) Sichert, J. A.; Tong, Y.; Mutz, N.; Vollmer, M.; Fischer, S.; Milowska, K. Z.; García Cortadella, R.; Nickel, B.; Cardenas-Daw, C.; Stolarczyk, J. K.; Urban, A. S.; Feldmann, J. Quantum Size Effect in Organometal Halide Perovskite Nanoplatelets. Nano Lett. 2015, 15, $6521-6527$

(12) Dou, L.; Wong, A. B.; Yu, Y.; Lai, M.; Kornienko, N.; Eaton, S. W.; Fu, A.; Bischak, C. G.; Ma, J.; Ding, T.; Ginsberg, N. S.; Wang, L.W.; Alivisatos, A. P.; Yang, P. Atomically Thin Two-Dimensional Organic-Inorganic Hybrid Perovskites. Science 2015, 349, 1518-1521.

(13) Akkerman, Q. A.; Motti, S. G.; Srimath Kandada, A. R.; Mosconi, E.; D’Innocenzo, V.; Bertoni, G.; Marras, S.; Kamino, B. A.; Miranda, L.; De Angelis, F.; Petrozza, A.; Prato, M.; Manna, L. Solution Synthesis Approach to Colloidal Cesium Lead Halide Perovskite Nanoplatelets with Monolayer-Level Thickness Control. J. Am. Chem. Soc. 2016, 138, 1010-1016.

(14) Shamsi, J.; Dang, Z.; Bianchini, P.; Canale, C.; Stasio, F. D.; Brescia, R.; Prato, M.; Manna, L. Colloidal Synthesis of Quantum Confined Single Crystal $\mathrm{CsPbBr}_{3}$ Nanosheets with Lateral Size Control up to the Micrometer Range. J. Am. Chem. Soc. 2016, 138, $7240-7243$ 
(15) Imran, M.; Di Stasio, F.; Dang, Z.; Canale, C.; Khan, A. H.; Shamsi, J.; Brescia, R.; Prato, M.; Manna, L. Colloidal Synthesis of Strongly Fluorescent $\mathrm{CsPbBr}_{3}$ Nanowires with Width Tunable Down to the Quantum Confinement Regime. Chem. Mater. 2016, 28, 64506454.

(16) Tong, Y.; Bladt, E.; Aygüler, M. F.; Manzi, A.; Milowska, K. Z.; Hintermayr, V. A.; Docampo, P.; Bals, S.; Urban, A. S.; Polavarapu, L.; Feldmann, J. Highly Luminescent Cesium Lead Halide Perovskite Nanocrystals with Tunable Composition and Thickness by Ultrasonication. Angew. Chem., Int. Ed. 2016, 55, 13887-13892.

(17) Yu, Y.; Zhang, D.; Kisielowski, C.; Dou, L.; Kornienko, N.; Bekenstein, Y.; Wong, A. B.; Alivisatos, A. P.; Yang, P. Atomic Resolution Imaging of Halide Perovskites. Nano Lett. 2016, 16, 75307535.

(18) Pan, Z. W.; Dai, Z. R.; Wang, Z. L. Lead Oxide Nanobelts and Phase Transformation Induced by Electron Beam Irradiation. Appl. Phys. Lett. 2002, 80, 309-311.

(19) Stanley, H. B.; Banerjee, D.; van Breemen, L.; Ciston, J.; Liebscher, C. H.; Martis, V.; Merino, D. H.; Longo, A.; Pattison, P.; Peters, G. W. M.; Portale, G.; Sen, S.; Bras, W. X-Ray Irradiation Induced Reduction and Nanoclustering of Lead in Borosilicate Glass. CrystEngComm 2014, 16, 9331-9339.

(20) Gnanavel, T.; Möbus, G. In-Situ Cobalt Nanocrystal Synthesis by Intense Electron Beams in Tem. J. Phys. Conf. Series 2012, 371, 012047.

(21) Sepulveda-Guzman, S.; Elizondo-Villarreal, N.; Ferrer, D.; Torres-Castro, A.; Gao, X.; Zhou, J. P.; Jose-Yacaman, M. In Situ Formation of Bismuth Nanoparticles through Electron-Beam Irradiation in a Transmission Electron Microscope. Nanotechnology 2007, 18,335604 .

(22) Pereira, W. d. S.; Andres, J.; Gracia, L.; San-Miguel, M. A.; da Silva, E. Z.; Longo, E.; Longo, V. M. Elucidating the Real-Time Ag Nanoparticle Growth on $\alpha-\mathrm{Ag}_{2} \mathrm{WO}_{4}$ During Electron Beam Irradiation: Experimental Evidence and Theoretical Insights. Phys. Chem. Chem. Phys. 2015, 17, 5352-5359.

(23) Tamou, Y.; Tanaka, S.-i. Formation and Coalescence of Tungsten Nanoparticles under Electron Beam Irradiation. Nanostruct. Mater. 1999, 12, 123-126.

(24) Smith, D. J.; McCartney, M. R.; Bursill, L. A. The ElectronBeam-Induced Reduction of Transition Metal Oxide Surfaces to Metallic Lower Oxides. Ultramicroscopy 1987, 23, 299-303.

(25) Knotek, M. L. Stimulated Desorption. Rep. Prog. Phys. 1984, 47, $1499-1561$.

(26) Gonzalez-Martinez, I. G.; Bachmatiuk, A.; Bezugly, V.; Kunstmann, J.; Gemming, T.; Liu, Z.; Cuniberti, G.; Rummeli, M. H. Electron-Beam Induced Synthesis of Nanostructures: A Review. Nanoscale 2016, 8, 11340-11362.

(27) El Mel, A.-A.; Molina-Luna, L.; Buffière, M.; Tessier, P.-Y.; Du, K.; Choi, C.-H.; Kleebe, H.-J.; Konstantinidis, S.; Bittencourt, C.; Snyders, R. Electron Beam Nanosculpting of Kirkendall Oxide Nanochannels. ACS Nano 2014, 8, 1854-1861.

(28) Egerton, R. F. Mechanisms of Radiation Damage in BeamSensitive Specimens, for Tem Accelerating Voltages between 10 and $300 \mathrm{Kv}$. Microsc. Res. Tech. 2012, 75, 1550-1556.

(29) Williams, D. B.; Carter, C. B. Transmission Electron Microscopy: A Textbook for Materials Science; Springer: New York, 2009.

(30) Raga, S. R.; Jung, M.-C.; Lee, M. V.; Leyden, M. R.; Kato, Y.; Qi, Y. Influence of Air Annealing on High Efficiency Planar Structure Perovskite Solar Cells. Chem. Mater. 2015, 27, 1597-1603.

(31) Wang, Y.; Li, X.; Sreejith, S.; Cao, F.; Wang, Z.; Stuparu, M. C.; Zeng, H.; Sun, H. Photon Driven Transformation of Cesium Lead Halide Perovskites from Few-Monolayer Nanoplatelets to Bulk Phase. Adv. Mater. 2016, 28, 10637-10643.

(32) Asoro, M. A.; Kovar, D.; Shao-Horn, Y.; Allard, L. F.; Ferreira, P. J. Coalescence and Sintering of Pt Nanoparticles: In Situ Observation by Aberration-Corrected HAADF STEM. Nanotechnology 2010, 21, 025701.
(33) Egerton, R. F. Beam-Induced Motion of Adatoms in the Transmission Electron Microscope. Microsc. Microanal. 2013, 19, 479-486.

(34) Elechiguerra, J. L.; Reyes-Gasga, J.; Yacaman, M. J. The Role of Twinning in Shape Evolution of Anisotropic Noble Metal Nanostructures. J. Mater. Chem. 2006, 16, 3906-3919.

(35) Tanaka, M.; Takeguchi, M.; Furuya, K. Behavior of Metal Nanoparticles in the Electron Beam. Micron 2002, 33, 441-446.

(36) Qian, J.; Xu, B.; Tian, W. A Comprehensive Theoretical Study of Halide Perovskites $\mathrm{ABX}_{3}$. Org. Electron. 2016, 37, 61-73.

(37) Vybornyi, O.; Yakunin, S.; Kovalenko, M. V. Polar-Solvent-Free Colloidal Synthesis of Highly Luminescent Alkylammonium Lead Halide Perovskite Nanocrystals. Nanoscale 2016, 8, 6278-6283.

(38) Jellicoe, T. C.; Richter, J. M.; Glass, H. F. J.; Tabachnyk, M.; Brady, R.; Dutton, S. E.; Rao, A.; Friend, R. H.; Credgington, D.; Greenham, N. C.; Böhm, M. L. Synthesis and Optical Properties of Lead-Free Cesium Tin Halide Perovskite Nanocrystals. J. Am. Chem. Soc. 2016, 138, 2941-2944.

(39) McLaughlan, S. D.; Evans, H. W. Production of Colloidal Calcium by Electron Irradiation of $\mathrm{CaF}_{2}$ Crystals. Phys. Status Solidi $B$ 1968, 27, 695-700.

(40) Schneider, C. A.; Rasband, W. S.; Eliceiri, K. W. Nih Image to Imagej: 25 Years of Image Analysis. Nat. Methods 2012, 9, 671-675. 